\title{
Carta e anarchia: una collezione di giornali anarchici della provincia di Pesaro e Urbino (1873 1922)
}

L'Archivio-biblioteca Enrico Travaglini di Fano ha promosso la ristampa anastatica completa dei giornali e numeri unici pubblicati dal movimento anarchico in provincia di Pesaro e Urbino, nel periodo compreso tra la prima Internazionale e l'avvento del fascismo. Attraverso questi giornali, dalla precaria vita editoriale e sempre sottoposti al controllo e alla censura delle autorità, emerge uno spaccato di vita politica di una provincia marchigiana a cavallo tra Otto e Novecento.

The Archive-Library Enrico Travaglini of Fano promoted the complete reprint of newspapers published by the anarchist movement in the province of Pesaro and Urbino, in the period between the first International and the rise of fascism. Through these papers, which had precarious editorial life and were always subject to control and censorship by the authorities, emerges an insight into the political life of a province of the Marche between the Nineteenth and Twentieth centuries.

\section{Il progetto bibliografico}

La sezione fanese dell'Internazionale fu una delle prime fondate nelle Marche, nel febbraio 1872, ed era presente alla Conferenza di Rimini del successivo 4 agosto, che sancì la rottura insanabile, su posizioni antiautoritarie, con il Consiglio generale ispirato alle tesi di Marx ed Engels e la nascita della Federazione italiana dell'Internazionale, ovvero del primo movimento anarchico organizzato. L'estate seguente, al congresso di Pietra la Croce (Ancona) del 10 agosto 1873, venne costituita la Federazione marchigiano-umbra. A dicembre iniziò le pubblicazioni 


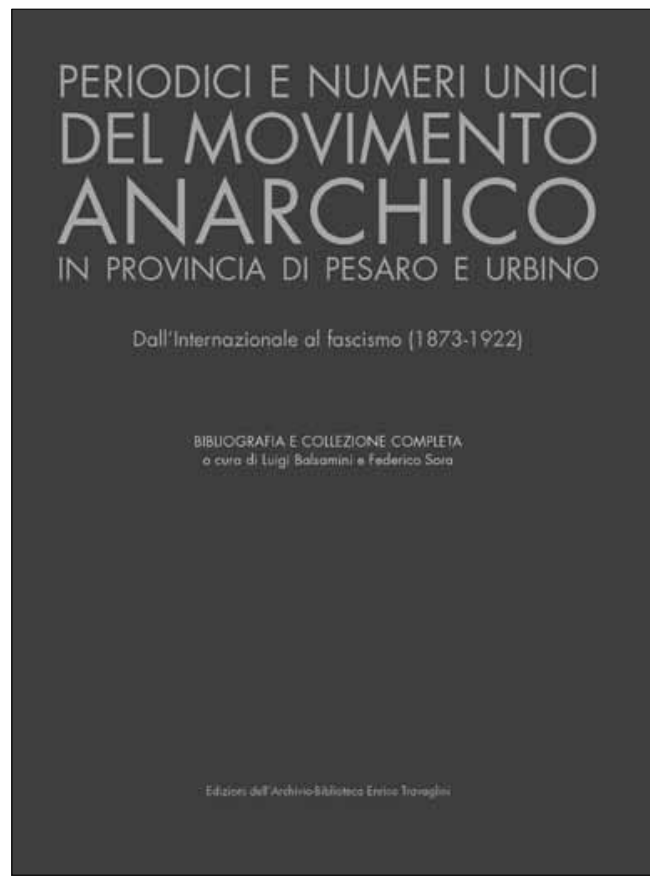

Copertina del volume

plementi. Nel dettaglio, le prime sono: "Il Comunardo" di Fano (1873-74), "L’Oppresso" di Pergola (1883), le tre serie del fanese "In Marcia" (1885-86; 1906; 1912-13), "Chi siamo!" di Pesaro (1890), "La Voce proletaria" di Fano (1919) e "La Frusta", stampata prima a Pesaro poi a Fano, con redazione e amministrazione trasferite da Pesaro, a Fano, a Sant'Angelo in Lizzola (1919-22).

L'elenco dei numeri unici e supplementi parte da "Commemorandum" di Pesaro del marzo 1890, passa, tra gli altri, per i fogli pubblicati dal Circolo di studi sociali di Fossombrone a fine Ottocento e i vari numeri collegati a "In marcia", per chiudersi con "La Se-
"Il Comunardo", «periodico socialistico». Con questo primo foglio internazionalista si apre la rassegna dei Periodici e numeri unici del movimento anarchico in provincia di Pesaro e Urbino, chiusa dal giornale di tendenza antiorganizzatrice "La Frusta", il cui ultimo numero uscì poche settimane prima della marcia su Roma.

Il volume, che ho avuto il piacere di curare insieme a Federico Sora, non si limita a un censimento bibliografico della pubblicistica libertaria, ma ne propone la ristampa anastatica, per un totale di oltre settecento pagine che riproducono integralmente otto serie di periodici e trentacinque numeri unici e sup-

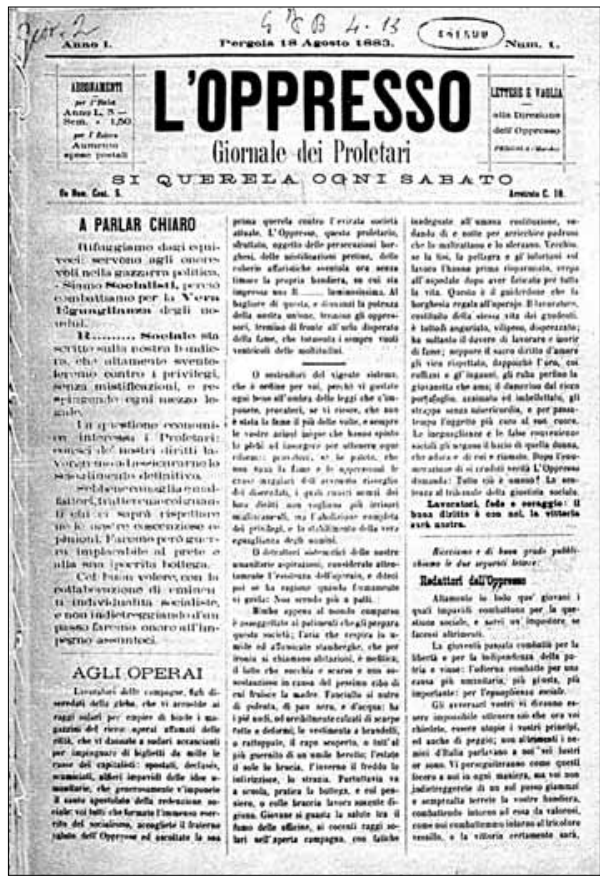

Un numero de "L'Oppresso" 
dia elettrica" del settembre 1921'. Ogni testata è corredata da un'approfondita scheda storico-bibliografica, che ne descrive dettagliatamente le caratteristiche formali, ne esamina la vita editoriale e si addentra in una rassegna degli articoli ospitati, collocandola nel contesto del movimento anarchico e della realtà politica locale.

Il trasferimento su copia anastatica è un'operazione che consente di coniugare efficacemente conservazione e consultazione. La stampa periodica presenta infatti una connaturata fragilità, dal momento che è prodotta per durare un breve periodo di tempo, un solo giorno nel caso dei quotidiani. E questa deteriorabilità è ancora più accentuata per via delle caratteristiche materiali della carta generalmente utilizzata in tipografia a partire da metà Ottocento. Il repertorio pubblicato dovrebbe garantire la possibilità di accedere alle fonti senza necessariamente maneggiare gli originali, pur nella consapevolezza che nessun surrogato, né la ristampa anastatica né tantomeno la copia digitale, possa sostituire il senso pieno di cui è portatore l'originale, non potendo supplire alla perdita dei significati connessi alla fisicità dell'oggetto originario, comprensivo di testo scritto e di supporto materiale. Il volume è stato editato in tiratura limitata di cento copie numerate dall'Archivio-biblioteca Enrico Travaglini di Fano, un istituto specializzato in storia e culture dei movimenti anarchici e libertari, che ha voluto suggellare con questa pubblicazione i suoi primi dieci anni di attività. Alcuni numeri inseriti nella raccolta sono posseduti in originale dalla stessa Biblioteca Travaglini, per la restante parte si è proceduto all'acquisizione in copia richiedendo riproduzioni a diversi archivi e biblioteche della provincia, alla Biblioteca nazionale centrale di Firenze $\mathrm{e}$, in misura minore, ad altri istituti in Italia e all'estero. Combinando quindi differenti provenienze è stato possibile ricomporre in un unico contenitore le serie complete dei periodici e la collezione di numeri unici.

\footnotetext{
1 Sono compresi nella bibliografia i numeri unici e i supplementi che si dichiarano esplicitamente tali, anche se si presentano in forma di fogli volanti. Questo l'elenco completo: "In marcia!..." (Fano-Pesaro, 23-24/10/1885, suppl. al n. 6); "Commemorandum" (Pesaro, 18/3/1890); "Chi siamo!" (Pesaro, 15/5/1890); "La Lotta elettorale politica" (Fossombrone, 6/11/1892); "18 Marzo" (Fossombrone, 18/3/1893, più ulteriore supplemento); "Risvegliamoci" (Urbino, 12/5/1894); "In marcia!..." (Fossombrone-Fano, 29/5/1894); "Vertenza” (Fossombrone, 29/11/1896); "Ciò che avviene nella nostra Congregazione di carità" (Fossombrone, 1/3/1903); "Verso la luce!" (Fano, 25/5/1903); "Rievocando i martiri dell'umanesimo!" (Fano, 11/11/1903); tre numeri unici sostitutivi di "In Marcia" (Fano, 22/06/1906, 30/6/1906, 7/7/1906) e un supplemento straordinario allo stesso giornale (Fano, 23/4/1906); "Contro le tenebre" (Fano, 14/7/1906); "Largo alla verità" (Fano, 13/10/1906); "Luce! Luce!" (Fano, dic. 1910); "Da più vasti orizzonti" (Fano, 17/12/1910); "Riformisti! Servi del capitalismo" (Fano, 23/12/1910); "I Pigmei" (Fano, 25/1/1911); due numeri che precedono la seconda serie di "In marcia" (Fano, 23/6/1912, 10/8/1912), un numero sostitutivo (Fano, 6/4/1913), uno che segue la fine delle pubblicazioni periodiche (Fano, 24/8/1913) e due supplementi: "Atti di eroismo?" (1912, non reperito) e "Per la verità e la giustizia" (Fano, 1913); "La Lotta elettorale" (Fano, 28/9/1913); "La Fiera elettorale" (Fano, 12/10/1913); "Marciamo!" (Fano, 18/3/1914); "II Diritto al lavoro" (Fano, 8/8/1920); tre supplementi a "La Frusta": "Documentando" (Fano, 15/9/1920), "Gruppo giovanile Sante Caserio" (Fano, 15/8/1921), "La Sedia elettrica” (Fano, 30/9/1921).
} 


\section{Periodici e numeri unici}

Attraverso la parola stampata, pur sempre affiancata alla comunicazione orale fondamentale in un contesto operaio scarsamente alfabetizzato, avveniva la trasmissione di valori e la formazione politico-culturale dei militanti. Ma il giornale era anche mezzo pratico di congiunzione delle attività sul territorio, dando informazione sull'attualità politica ed economica, notizie di scioperi e agitazioni, cronache locali, per cui rappresenta, oggi, una fonte primaria per la storiografia sul movimento libertario. La presenza di una pubblicazione periodica regolare era spesso un segnale della forza del movimento in una determinata congiuntura, tanto più in una dimensione provinciale dove la distribuzione della stampa marcava una presenza politica ed era il principale veicolo per sostenere le idee e farle prevalere su quelle avversarie, quasi un prolungamento della piazza cittadina. L'uscita del giornale, del proprio giornale, era infatti un mezzo di identificazione e anche di orgoglio, che rafforzava l'appartenenza di classe e stimolava la continuità dell'impegno. Rappresentava, inoltre, un punto di riferimento e di legame con la propria comunità d'origine per quanti avevano preso la via dell'esilio.

Diverse copie venivano distribuite infatti negli ambienti dell'emigrazione libertaria all'estero, in particolare Svizzera, Francia e soprattutto Stati Uniti, anche se il raggio di diffusione della stampa raccolta nel volume rimaneva in gran parte locale e provinciale, con sconfinamenti abituali nelle limitrofe zone dell'anconetano e della Romagna. Da questo circondario arrivavano la maggior parte delle corrispondenze e le sottoscrizioni economiche. L'elenco di quest'ultime, al quale veniva tradizionalmente dedicata la colonna di chiusura, restituisce un vero e proprio spaccato di vita sociale, con l'«avanzo di bicchierata» che il gruppo di lavoratori mandava al giornale o qualche lira raccolta tra compagni «inneggiando alla Rivoluzione Sociale», a volte spartita anche con gli organi di stampa socialisti e repubblicani.

Non sempre è possibile determinare la tiratura raggiunta dalle varie testate e numeri unici, che indicativamente era compresa tra le 3-400 copie de "Il Comunardo" del 1873-74 e le 4.500 toccate da "La Frusta" nel 1922, dopo un inizio sulle 2.500 e un assestamento intorno alle 3.000-3.500 raggiunto dallo stesso periodico nel 1920. Un tratto comune di questi giornali era la vita editoriale, quasi sempre molto breve: limitata a poche annate, ma più spesso addirittura compresa nell'arco di qualche mese. La periodicità dichiarata, in genere settimanale o quindicinale, difficilmente manteneva regolarità, per cause legate sia alle vicissitudini personali dei redattori sia alla strutturale precarietà finanziaria. 
Le pubblicazioni prese in considerazione erano sì espressione di un gruppo redazionale più o meno allargato di giornalisti non professionisti, ma il più delle volte erano legate anche alla forza trainante di singole personalità, che esercitavano la loro impronta nella direzione e alle cui biografie le vicende editoriali finivano per essere sostanzialmente sovrapponibili (tra questi Espartero Bellabarba, Ettore Antonelli, Giovanni Geronzi, Enrico Travaglini, Casimiro Accini, Giobbe Sanchini). Alcuni collaboratori locali divennero firme abbastanza stabili, che si affiancavano a contributi inediti provenienti anche da nomi noti nel panorama dell' anarchismo nazionale, come, fra gli altri, l'avvocato Francesco Saverio Merlino nella prima serie di "In marcia" (1885-86), la scrittrice Leda Rafanelli nella seconda (1906), l'anarchico senigalliese Ottorino Manni nelle pubblicazioni novecentesche, l'antiorganizzatore Luigi Galleani per "La Frusta".

\section{Controllo e censura}

I giornali del movimento anarchico, di cui abbiamo potuto ricostruire la raccolta completa recuperando i pochissimi numeri superstiti, non ebbero affatto vita facile. Su di essi e sui loro redattori era infatti costante la sorveglianza poliziesca attenta a reprimere la propaganda sovversiva, che si manifestava sia come censura preventiva, sia imbastendo processi per i reati di opinione ravvisati negli articoli pubblicati. Il quadro legislativo specifico all'interno del quale ci si muoveva era dato dall'editto sulla stampa promulgato da Carlo Alberto il 26 marzo 1848, rimasto in vigore nell'ordinamento dello stato unitario pur con le varie modifiche successivamente apportate. L'atteggiamento nei confronti della stampa, delle sue libertà e restrizioni, era quanto mai ambiguo: «la stampa sarà libera, ma soggetta a leggi repressive», recitava il proclama costituzionale dell' 8 febbraio.

L'editto segnò il passaggio da un regime di censura preventiva a un sistema repressivo, riconoscendo il diritto di pubblicare giornali senza bisogno di autorizzazione, ma a seguito di una semplice dichiarazione. All'atto pratico però, le modalità per operare censure anche preventive rimanevano ampiamente a disposizione delle autorità che, ricevuto il primo esemplare della pubblicazione, potevano ordinare il sequestro di tutte le copie e delle matrici di stampa inviando gli agenti in tipografia, salvo in qualche caso farli arrivare fuori tempo massimo e non trovare più nulla in giacenza. In sostanza, se veniva ammesso il principio della libertà di stampa, in base al quale pubblicare un giornale non era più un privilegio concesso dal sovrano ma un diritto del cittadino, immediatamente dopo lo si negava lasciando ampi margini di discrezionalità alla sua repressione da parte delle autorità di polizia e della magistratura. 


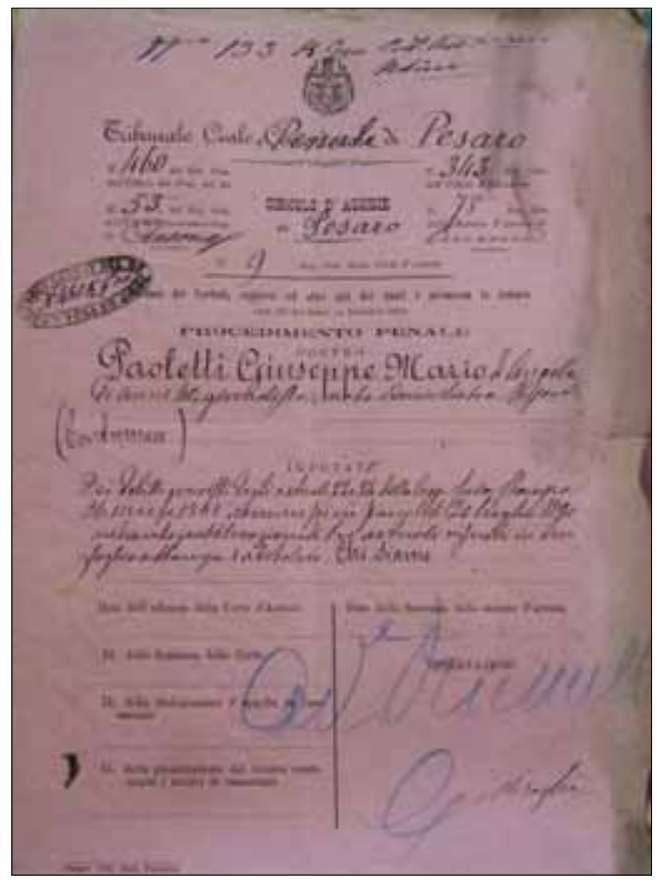

Un esempio di procedimento penale contro un "giornalista» anarchico
Pertanto procure, tribunali e Corte d'assise si occupavano a pieno regime di stampa sovversiva, compilando interi fascicoli di verbali, mandati di comparizione, interrogatori, certificati penali, istruttorie e sentenze, e ricopiando minuziosamente a ogni passaggio giudiziario i brani degli articoli che avrebbero "abusato" della libertà d'espressione: presso l'Archivio di stato di Pesaro sono stati rintracciati ben trantaquattro faldoni riguardanti processi penali contro i responsabili dei giornali anarchici locali. La conseguenza non voluta di queste meticolose attenzioni è che i fascicoli di polizia e dei tribunali conservati negli archivi di stato sono oggi un fecondo terreno di ricerca per ricostruire le storie editoriali delle testate, nonché per reperirne esemplari giunti in seguito a sequestri. Il controllo sulla stampa sovversiva si traduceva quindi in processi e condanne che coinvolgevano principalmente i gerenti responsabili delle varie testate e spesso erano il fattore determinante che conduceva alla sospensione delle pubblicazioni. Nel caso di Enrico Travaglini, l'accumularsi di circa trenta mesi inflittigli dai tribunali per una lunga serie di reati dovuti ai suoi articoli (per non aver ottemperato alle leggi sulla stampa, per incitamento all'odio tra le classi, apologia di regicidio, istigazione a delinquere, oltraggio e diffamazione ecc.) lo costrinse a espatriare e rifugiarsi negli Stati Uniti. Al suo nome è ora intitolato l'Archivio-bilioteca che ha promosso la pubblicazione di questo volume e che, prossimamente, farà seguire un'analoga iniziativa editoriale, già in cantiere, per manifesti, fogli volanti e opuscoli libertari locali. 


\section{Risorse}

Biblioteca Travaglini:

http://www.bibliotecaliberopensiero.it

Istituto di storia contemporanea della Provincia di Pesaro e Urbino: http://www.bobbato.it

Rete Storia Marche 900:

http://www.storiamarche900.it 
